\title{
IRREGULAR BEHAVIOUR OF CLASS NUMBERS AND EULER-KRONECKER CONSTANTS OF CYCLOTOMIC FIELDS: THE LOG LOG LOG DEVIL AT PLAY
}

\author{
PIETER MOREE
}

\begin{abstract}
Kummer (1851) and, many years later, Ihara (2005) both posed conjectures on invariants related to the cyclotomic field $\mathbb{Q}\left(\zeta_{q}\right)$ with $q$ a prime. Kummer's conjecture concerns the asymptotic behaviour of the first factor of the class number of $\mathbb{Q}\left(\zeta_{q}\right)$ and Ihara's the positivity of the Euler-Kronecker constant of $\mathbb{Q}\left(\zeta_{q}\right)$ (the ratio of the constant and the residue of the Laurent series of the Dedekind zeta function $\zeta_{\mathbb{Q}\left(\zeta_{q}\right)}(s)$ at $s=1$ ). If certain standard conjectures in analytic number theory hold true, then one can show that both conjectures are true for a set of primes of natural density 1 , but false in general. Responsible for this are irregularities in the distribution of the primes.

With this survey we hope to convince the reader that the apparently dissimilar mathematical objects studied by Kummer and Ihara actually display a very similar behaviour.
\end{abstract}

\section{INTRODUCTION}

Making conjectures in analytic prime number theory is a notoriously dangerous endeavoun 1 certainly if the basis for this is mostly of numerical nature. The danger lies in the fact that computers can barely spot log log terms and are certainly blind to the log log log terms that frequently occur. The presence of such terms can result in the conjecture being false on very thin subsequences. Celebrated examples are the $\pi(x)<\operatorname{Li}(x)$ conjecture and the Mertens conjecture that $\left|\sum_{n \leq x} \mu(n)\right|<\sqrt{x}$ for $n \geq 1$ (for notation see Section 2.1). Both of them are false, but true up to gigantic values of $x$. A way out of the danger zone is to change "for all" to some slightly weaker statement. However, this requires a substantial theoretical insight into the conjecture.

2010 Mathematics Subject Classification. 11N37, 11Y60, 11M20.

${ }^{1}$ In fact, the title of this paper ends with a question mark. Since it is considered very bad style to have it in the title of a paper, this footnote might be a better place. Not putting the question mark would go against the moral of this paper. 
Here we present two further conjectures (due to Kummer and Ihara respectively) where the phenomena indicated above also seem to arise. The final verdict on them is still open but, assuming some standard conjectures from analytic number theory, they are false on some very thin sequences of primes due to irregularities in the distribution of the primes. At a first glance the two conjectures look unrelated. However, they are both connected with the distribution of special $L$-values and the results and conjectures we present on them are strikingly similar $2^{2}$.

In the remaining part of the introduction we formulate the conjectures (after stating some background material) and discuss how they are related to special $L$-values. In the rest of the paper we discuss results and related conjectures.

Although results from various papers are mentioned in this survey, our main inspiration are Ford, Luca and Moree [7] for the EulerKronecker constant and Granville [11] for Kummer's conjecture. EulerKronecker constants for non-quadratic fields were put on the mathematical map mainly thanks to the efforts of Ihara [15, 16, 17].

1.1. The Euler-Kronecker constant for number fields. For a number field $K$ we can define, for $\operatorname{Res}>1$, the Dedekind zeta function by

$$
\zeta_{K}(s)=\sum_{\mathfrak{a}} \frac{1}{N \mathfrak{a}^{s}}=\prod_{\mathfrak{p}} \frac{1}{1-N \mathfrak{p}^{-s}} .
$$

Here, $\mathfrak{a}$ runs over the non-zero ideals in $\mathcal{O}_{K}$, the ring of integers of $K, \mathfrak{p}$ runs over the prime ideals in $\mathcal{O}_{K}$ and $N \mathfrak{a}$ is the norm of $\mathfrak{a}$. It is known that $\zeta_{K}(s)$ can be analytically continued to $\mathbb{C}-\{1\}$, and that at $s=1$ it has a simple pole and residue $\alpha_{K}$. The prime ideals having prime norm are of particular importance as they are the cause for this pole.

After a suitable normalisation with gamma factors, one obtains from $\zeta_{K}(s)$ a function $\tilde{\zeta}_{K}(s)$ satisfying the functional equation

$$
\tilde{\zeta}_{K}(s)=\tilde{\zeta}_{K}(1-s)
$$

Since $\tilde{\zeta}_{K}(s)$ is entire of order 1 , one has the following Hadamard product factorization:

$$
\tilde{\zeta}_{K}(s)=\tilde{\zeta}_{K}(0) e^{\beta_{K} s} \prod_{\rho}\left(1-\frac{s}{\rho}\right) e^{\frac{s}{\rho}},
$$

with $\beta_{K} \in \mathbb{C}$ and where $\rho$ runs over the zeros of $\zeta_{K}(s)$ in the critical strip.

\footnotetext{
${ }^{2}$ The similarity was first noted by Andrew Granville, see acknowledgment.
} 
Around $s=1$ we have the Laurent expansion

$$
\zeta_{K}(s)=\frac{\alpha_{K}}{s-1}+c_{K}+c_{1}(K)(s-1)+c_{2}(K)(s-1)^{2}+\ldots
$$

The constant $\gamma_{K}=c_{K} / \alpha_{K}$ is called the Euler-Kronecker constant in Ihara [15] and Tsfasman [35. In particular, we have $c_{\mathbb{Q}}=\gamma=$ $0.57721566 \ldots$, the Euler-Mascheroni constant, see e.g. Lagarias [20] for a wonderful survey of related material. In case $K$ is imaginary quadratic, the well-known Kronecker limit formula expresses $\gamma_{K}$ in terms of special values of the Dedekind $\eta$-function.

An alternative formula for $\gamma_{K}$ is given by

$$
\gamma_{K}=\lim _{s \downarrow 1}\left(\frac{\zeta_{K}^{\prime}(s)}{\zeta_{K}(s)}+\frac{1}{s-1}\right)
$$

which shows that $\gamma_{K}$ is the constant part in the Laurent series of the logarithmic derivative of $\zeta_{K}(s)$. Using the Hadamard factorization (1) one can relate $\gamma_{K}$ to the sum of the reciprocal zeros of $\zeta_{K}(s)$, cf. [7, p. 1452]. Indeed, in a lot of the literature the logarithmic derivative of the right hand side of (1) is the starting point in studying $\gamma_{K}$. The main tool of Ihara, cf. [15, p. 411], is an "explicit" formula for the prime function

$$
\Phi_{K}(x)=\frac{1}{x-1} \sum_{N \mathfrak{p}^{k} \leq x}\left(\frac{x}{N \mathfrak{p}^{k}}-1\right) \log N \mathfrak{p}, x>1,
$$

relating it to the zeros of $\zeta_{K}(s)$.

Given any Dirichlet series $L(s)$ with a pole at $s=1$, we can define its Euler-Kronecker constant as the constant part in the Laurent series of its logarithmic derivative (if this constant exists). In Moree [25] this is considered in case when $S$ is a multiplicative set of integers (that is, for coprime integers $m$ and $n$ one has $m n \in S$ if and only if both $m$ and $n$ are in $S$ ) and $L_{S}(s)=\sum_{n \in S} n^{-s}$ is its associated Dirichlet series.

Another alternative formula for $\gamma_{K}$ is given by

$$
\gamma_{K}=\lim _{x \rightarrow \infty}\left(\log x-\sum_{N \mathfrak{p} \leq x} \frac{\log N \mathfrak{p}}{N \mathfrak{p}-1}\right) .
$$

This result is due to de la Vallée-Poussin (1896) in case $K=\mathbb{Q}$ and can be easily generalized to other number fields and settings, cf. [12, 13].

Ihara [15, Theorem 1 and Proposition 3] proved that GRH (Conjecture 6 below) implies that there are absolute constants $c_{1}, c_{2}>0$ such that

$$
-c_{1} \log \left|d_{K}\right|<\gamma_{K}<c_{2} \log \log \left|d_{K}\right|
$$


where $d_{K}$ denotes the discriminant $K / \mathbb{Q}$. Tsfasman [35] showed that the above lower bound is sharp, namely, assuming GRH he proved that

$$
\liminf \frac{\gamma_{K}}{\log \left|d_{K}\right|} \geq-0.13024 \ldots,
$$

where we range over the number fields $K$ with $\left|d_{K}\right| \rightarrow \infty$. Later Badzyan [3] proved that one can take $c_{1}=(1-1 / \sqrt{5}) / 2 \approx 0.276393$. It is an open problem whether this is sharp.

1.2. The Euler-Kronecker constant for cyclotomic fields. It is a natural question to ask how the Euler-Kronecker constant varies over families of number fields such as quadratic fields and (maximal) cyclotomic fields. After quadratic fields, cyclotomic fields have been most intensively studied (see [21, 36] for book length treatments). Many of the associated quantities of a cyclotomic field $\mathbb{Q}\left(\zeta_{m}\right)$ are explicitly known. Relevant examples for us are their ring of integers, $\mathbb{Z}\left[\zeta_{m}\right]$, and their discriminant

$$
d_{\mathbb{Q}\left(\zeta_{n}\right)}=(-1)^{\varphi(n) / 2} n^{\varphi(n)} \prod_{p \mid n} p^{-\frac{\varphi(n)}{(p-1)}} .
$$

Moreover, the splitting of a rational prime $p$ into prime ideals in $\mathbb{Z}\left[\zeta_{m}\right]$ of a cyclotomic field follows an easy pattern, see e.g. [31, Theorem 4.16], which we recall here.

For $p$ a prime not diving the integer $m$, we define $\operatorname{ord}_{p}(m)$ to be the (multiplicative) order of $p$ in $(\mathbb{Z} / m \mathbb{Z})^{*}$

Lemma 1 (Cyclotomic reciprocity law). Let $K=\mathbb{Q}\left(\zeta_{m}\right)$. If the prime $p$ does not divide $m$ and $f=\operatorname{ord}_{p}(m)$, then the principal ideal $p \mathcal{O}_{K}$ factorizes as $\mathfrak{p}_{1} \cdots \mathfrak{p}_{g}$ with $g=\varphi(m) / f$ and all $\mathfrak{p}_{\mathfrak{i}}$ are distinct and of degree $f$.

However, if $p$ divides $m, m=p^{a} m_{1}$ with $p \nmid m_{1}$ and $f=\operatorname{ord}_{p}\left(m_{1}\right)$, then $p \mathcal{O}_{K}=\left(\mathfrak{p}_{1} \cdots \mathfrak{p}_{g}\right)^{e}$ with $e=\varphi\left(p^{a}\right), g=\varphi\left(m_{1}\right) / f$ and all $\mathfrak{p}_{\mathfrak{i}}$ are distinct and of degree $f$.

For notational convenience we will write $\gamma_{m}$ instead of $\gamma_{\mathbb{Q}\left(\zeta_{m}\right)}$. Our main focus is on the case where $m=q$ is a prime (unless specified otherwise, $m$ denotes a positive integer and $p$ and $q$ primes). Then we have

$$
\zeta_{\mathbb{Q}\left(\zeta_{q}\right)}(s)=\zeta(s) \prod_{\chi \neq \chi_{0}} L(s, \chi),
$$

where $\chi$ ranges over the non-trivial characters modulo $q$, leading to

$$
\gamma_{q}=\gamma+\sum_{\chi \neq \chi_{0}} \frac{L^{\prime}(1, \chi)}{L(1, \chi)}
$$


Thus the behaviour of $\gamma_{q}$ is related to that of $L(s, \chi)$ and $L^{\prime}(s, \chi)$ at $s=1$. (Here and in the rest of the paper we often use the fundamental fact that $L(1, \chi) \neq 0$.)

Let $\mathbb{Q}\left(\zeta_{m}\right)^{+}$denote the maximal real subfield of $\mathbb{Q}\left(\zeta_{m}\right)$ and $\gamma_{m}^{+}$its Euler-Kronecker constant. In that case we find

$$
\zeta_{\mathbb{Q}\left(\zeta_{q}\right)^{+}}(s)=\zeta(s) \prod_{\substack{\chi \neq \chi_{0} \\ \chi(-1)=1}} L(s, \chi) .
$$

Logarithmic differentiation of the latter product identity then yields

$$
\gamma_{q}^{+}=\gamma+\sum_{\substack{\chi \neq \chi_{0} \\ \chi(-1)=1}} \frac{L^{\prime}(1, \chi)}{L(1, \chi)}
$$

1.3. Ihara's conjecture. Ihara made a conjecture on $\gamma_{m}$ based on numerical observations for $m \leq 8000$, which we here only formulate in case $m=q$ is prime.

Conjecture 1 (Ihara's conjecture [16]). Let $q \geq 3$ be a prime.

1) $\gamma_{q}>0$ ('very likely');

2) For fixed $\varepsilon>0$ and $q$ sufficiently large we have

$$
\frac{1}{2}-\varepsilon \leq \frac{\gamma_{q}}{\log q} \leq \frac{3}{2}+\varepsilon
$$

The most extensive computations on $\gamma_{q}$ to date were carried out by Ford et al. [7].

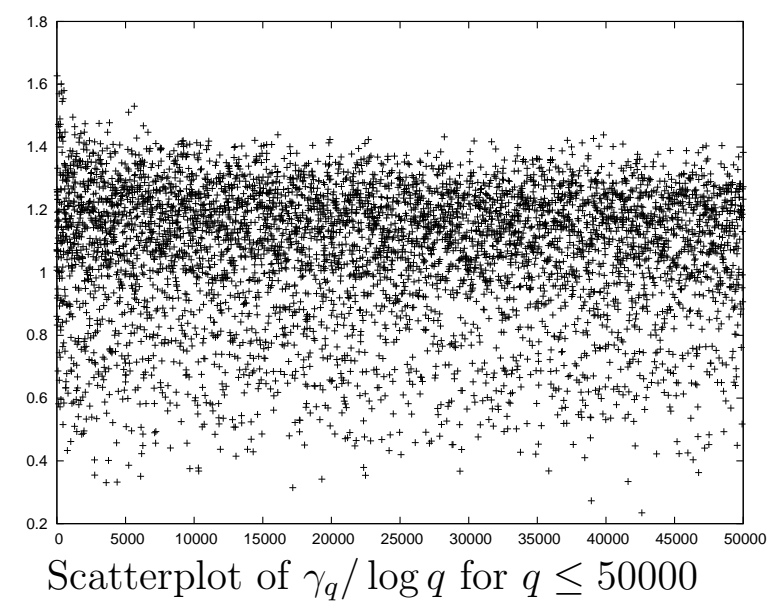

The largest value of $\gamma_{q} / \log q$ among $q \leq 30000$ equals $1.626 \ldots$ and occurs at $q=19$. The smallest is $0.315 \ldots$ and occurs at $q=17183$. 
It is a consequence of (5), (6) and Badzyan's result mentioned above that, under GRH, there exists a constant $c_{2}^{\prime}>0$ such that

$$
-(1-1 / \sqrt{5}) q(\log q) / 2<\gamma_{q}<c_{2}^{\prime} \log q .
$$

Ihara [15] showed that $\gamma_{q} \leq(2+o(1)) \log q$ assuming ERH (Conjecture 5 below). The lower bound in (11) turns out to be very weak. Ihara et al. [18] proved that for any $\varepsilon>0$ one has $\left|\gamma_{q}\right|=O_{\varepsilon}\left(q^{\varepsilon}\right)$ and, under GRH, $\left|\gamma_{q}\right|=O\left(\log ^{2} q\right)$. We will see in Section 3 that these bounds can be sharpened considerably.

1.4. Kummer's conjecture. Let $h_{1}(q)$ be the ratio of the class number $h(q)$ of $\mathbb{Q}\left(\zeta_{q}\right)$ and the class number $h_{2}(q)$ of its maximal real subfield $\mathbb{Q}\left(\zeta_{q}+\zeta_{q}^{-1}\right)$, that is, $h_{1}(q)=h(q) / h_{2}(q)$. Kummer proved that this is an integer. It is now called the first factor of the class number of $h(q)$.

In 1851 Kummer [19] published a review of the main results that he and others had discovered about cyclotomic fields. In this elegant report he made the following conjecture.

Conjecture 2 (Kummer's conjecture [19]). Put

$$
G(q)=\left(\frac{q}{4 \pi^{2}}\right)^{\frac{q-1}{4}} \text { and } r(q)=\frac{h_{1}(q)}{G(q)} .
$$

Then asymptotically $r(q)$ tends to 1 .

In fact he claimed to have a proof that he would publish later together with further developments (but never did). Kummer himself laboriously computed $r(q)$ for $q<100$. This was extended over time by many authors, more recently by Shokrollahi [33]. He showed that the largest value of $r(q)$ among $q \leq 10000$ equals $1.556562 \ldots$ and occurs at $q=5231$. The smallest is $0.642429 \ldots$ and occurs at $q=3331$.

In 1949 Ankeny and Chowla [1, 2] made some progress by showing that

$$
\log r(q)=o(\log q)
$$

Siegel [34], who was unaware of the earlier work of Ankeny and Chowla, proved a weaker version of (12) and was one of the first to cast doubt on the truth of Kummer's conjecture. From (12) we infer that

$$
\log h_{1}(q) \sim \frac{q}{4} \log q
$$

and thus that there are only finitely many primes $q$ such that $\mathbb{Q}\left(\zeta_{q}\right)$ has class number one. This was made effective by Masley and Montgomery [22], who showed that $|\log r(q)|<7 \log q$ for $q>200$, which is strong enough to establish Kummer's conjecture that $h_{1}(q)=1$ if and only if 
$q \leq 19$. This result is their key ingredient in determining all cyclotomic fields having class number 1. In proving their upper bound, Masley and Montgomery used zero-free regions of $L$-functions. This idea was refined by Puchta [32], with a further improvement by Debaene [5], to obtain an upper bound for $\log r(q)$ that depends on a Siegel zero, if it exists.

Not surprisingly $h_{1}$ is eventually monotonic; however, no beginning prime is yet known. In this direction Fung et al. [9, Theorem 1] showed that if $E$ is an elliptic curve over $\mathbb{Q}$ for which the associated $L$-function has a zero of order at least 6 in $s=1$, then it is possible to find an explicit prime $q_{0}$ for which $h_{1}\left(q_{2}\right)>h_{1}\left(q_{1}\right)$, whenever $q_{2}>q_{1} \geq q_{0}$. It is believed that one can take $q_{0}=19$.

Just like $\gamma_{q}$ in (8), $h_{1}(q)$ is also related to special values of Dirichlet $L$-series. Hasse [14] showed that

$$
r(q)=\frac{h_{1}(q)}{G(q)}=\prod_{\chi(-1)=-1} L(1, \chi),
$$

where the product is over all the odd characters modulo $q$. It follows from this, (7) and (9) that

$$
r(q)=\lim _{s \downarrow 1} \frac{\zeta_{\mathbb{Q}\left(\zeta_{q}\right)}(s)}{\zeta_{\mathbb{Q}\left(\zeta_{q}\right)^{+}}(s)} .
$$

Indeed, using the definition of Euler-Kronecker constant we find the Taylor series expansion around $s=1$

$$
\frac{\zeta_{\mathbb{Q}\left(\zeta_{q}\right)}(s)}{\zeta_{\mathbb{Q}\left(\zeta_{q}\right)^{+}}(s)}=r(q)\left(1+\left(\gamma_{q}-\gamma_{q}^{+}\right)(s-1)+O_{q}\left((s-1)^{2}\right)\right),
$$

involving both of the main actors of this survey ${ }^{3}$.

1.5. Similarities between the two conjectures. The remaining part of this survey will make clear that the quantities

$$
\frac{\gamma_{q}}{\log q} \quad \text { and } \quad 1-2|\log r(q)|
$$

have very similar analytical properties. Indeed, this analogy implies that the Euler-Kronecker analogue of the Kummer conjecture is that asymptotically

$$
\gamma_{q} \sim \log q
$$

The numerical computations mentioned above suggest that both quantities in (15) are bounded, whereas if one believes in some standard conjectures in analytic number theory (delineated in the next section),

\footnotetext{
${ }^{3}$ I have not come across this formula in the literature.
} 
they can be sporadically very negative. Various researchers in this area believe that it is the $\log \log \log$ devil that ruins both the Kummer and Ihara conjecture (see Section 5).

\section{Preliminaries}

2.1. Standard conjectures used. The results we are going to present depend on some standard conjectures on the prime distribution that we briefly recall in this section.

Let $\mathcal{A}=\left\{a_{1}, \ldots, a_{s}\right\}$ be a set consisting of $s$ distinct natural numbers. We define

$$
m(\mathcal{A})=\sum_{k=1}^{s} \frac{1}{a_{i}}
$$

The set $\mathcal{A}$ is said to be admissible if there does not exist a prime $p$ such that $p \mid n \prod_{i=1}^{s}\left(a_{i} n+1\right)$ for every $n \geq 1$. Note that if there is such a prime factor $p$, then $p \leq s+1$. The sequence $\{a(i)\}_{i=1}^{\infty}=$ $\{2,6,8,12,18,20,26,30,32, \ldots\}$ has the property that any finite sub sequence is an admissible set. It is called "the greedy sequence of prime offsets" and is sequence A135311 in the Online Encyclopedia of Integer Sequences (OEIS).

Conjecture 3 (Hardy-Littlewood). Suppose $\mathcal{A}=\left\{a_{1}, \ldots, a_{s}\right\}$ is an admissible set. Then the number of primes $n \leq x$ such that the integers $a_{1} n+1, \ldots, a_{s} n+1$ are all prime is $\gg_{\mathcal{A}} x \log ^{-s-1} x$.

Actually, the full Hardy-Littlewood conjecture gives an asymptotic, rather than a lower bound. It is this full version that was used by Croot and Granville [4] to study how many primes $q \leq x$ satisfy $r(q)=$ $\alpha+o(1)$, with $\alpha>0$ and fixed.

As usual, we let $\pi(x ; d, a)$ denote the number of primes $p \leq x$ satisfying $p \equiv a(\bmod d), \pi(x)$ the prime counting function, $\operatorname{Li}(x)$ the logarithmic integral and $\mu$ the Möbius function.

Conjecture 4 (Elliott-Halberstam). For any $\varepsilon>0$ and $C>0$ we have

$$
\sum_{k<x^{1-\varepsilon}} \max _{(l, k)=1} \max _{y \leq x}\left|\pi(y ; k, l)-\frac{\operatorname{Li}(x)}{\varphi(k)}\right| \ll_{\varepsilon, C} \frac{x}{(\log x)^{C}} .
$$

Conjecture 5 (Extended Riemann Hypothesis). Every Dirichlet series $L(s, \chi)$ satisfies the Riemann Hypothesis.

Conjecture 6 (Generalized Riemann Hypothesis). Every Dedekind zeta function $\zeta_{K}(s)$ satisfies the Riemann Hypothesis. 
In places where one uses GRH for a general number field, for a cyclotomic number field ERH suffices, as their Dedekind zeta function decomposes as a product of Dirichlet $L$-series, cf. (7).

For most results quoted below a weaker form of these conjectures suffices. For reasons of brevity we leave out the details and refer the reader to the original publications. Also for brevity we will refer to the above conjectures by the abbreviations HL, EH, ERH and GRH, respectively.

2.2. The distribution of $m(\mathcal{A})$. Crucial for obtaining results on both the Kummer and the Ihara conjecture is an understanding of the distribution of $m(\mathcal{A})$ as $\mathcal{A}$ ranges over the admissible sets. We put $\mathcal{M}=\{m(\mathcal{A}): \mathcal{A}$ is admissible $\}$ and let $\overline{\mathcal{M}}$ be the closure of $\mathcal{M}$, that is, the set of limit points of sequences of elements of $\mathcal{M}$ that do converge.

Granville showed that the following 1988 conjecture by Erdős is tru£ $\AA^{4}$.

Theorem 1 (Granville [11]). There is a sequence of admissible sets $\mathcal{A}_{1}, \mathcal{A}_{2}, \ldots$ such that $\lim _{j \rightarrow \infty} m\left(\mathcal{A}_{j}\right)=\infty$.

Corollary 1. We have $\overline{\mathcal{M}}=[0, \infty]$.

Proof. Given any $x>0$ and $\delta>0$, there is an admissible set $\mathcal{A}$ with $m(\mathcal{A})>x$ consisting of integers all $>1 / \delta$. As any subset of an admissible set is also admissible, there is a subset $\mathcal{A}^{\prime}$ of $\mathcal{A}$ with $\left|m\left(\mathcal{A}^{\prime}\right)-x\right|<\delta$.

Another issue is that of finding admissible subsets $\mathcal{A} \subseteq[1, x]$ having large $m(\mathcal{A})$. In this direction Granville proved the following result.

Proposition 1 (Granville [11]).

1) For any sufficiently large $x$ there is an admissible set $\mathcal{A}$, which is a subset of $[1, x]$, with $m(\mathcal{A}) \geq(1+o(1)) \log \log x$.

2) There exists a constant $c>0$ such that if $\mathcal{A}$ is an admissible subset of $[1, x]$, then $m(\mathcal{A}) \leq c \log \log x$.

Granville believes one can take $c=1+\varepsilon$, for any $\varepsilon>0$, provided that $x$ is sufficiently large. If true, this would imply that part 1 is best possible.

\footnotetext{
${ }^{4}$ The authors of 7], unaware of Granville's work and the fact that they were dealing with a(n) (ex-)conjecture of Erdős, gave a short different proof using a 1961 paper of... Erdős [6] himself!
} 


\section{The COnstants $\gamma_{q}$ : RESUlts And CONJECTURES}

On applying (4) and Lemma 1 we obtain

$$
\gamma_{q}=-\frac{\log q}{q-1}-S(q)-\lim _{x \rightarrow \infty}\left(\log x-(q-1) \sum_{\substack{p \leq x \\ p \equiv 1(\bmod q)}} \frac{\log p}{p-1}\right),
$$

where

$$
S(q)=(q-1) \sum_{\substack{p \neq q \\ \operatorname{ord}_{p}(q) \geq 2}} \frac{\log p}{p^{\operatorname{ord}_{p}(q)}-1} .
$$

By Lemma 1 the only rational primes splitting into prime ideals of prime norm are $q$ and all the primes $p \equiv 1(\bmod q)$. They are responsible for the first, respectively third term on the right hand side of (16). The term $S(q)$ is the contribution of the prime ideals lying above the remaining rational primes. Using estimates for linear forms in logarithms, it can be shown that $S(q) \leq 45$ and even that for any fixed $\varepsilon>0$ we have $S(q)<\varepsilon$ for $(1+o(1)) \pi(x)$ primes $q \leq x[7$, Theorem $3]$. Since, as we will see, $\gamma_{q}$ has normal order $\log q$, it follows that the first two terms in (16) are error terms.

The idea now is to approximate $\gamma_{q}$ by choosing a suitable value for $x$ in (16). In principle one wants to have $x$ small, but the irregularities in the distribution of the primes do not allow us to take $x$ too small. The Bombieri-Vinogradov theorem allows us to take $x=q^{2+\delta}$ for any $\delta>0$ with the possible exception of a thin set of primes. Using the BrunTitchmarsh inequality one can bring this down to $x=q^{2}$. Likewise, assuming EH one can go down to $x=q^{1+\delta}$. This approach leads to the following result.

Lemma 2 (Ford et al. [7]). Given $r>1$ write

$$
E_{r}(q)=\gamma_{q}-r \log q+q \sum_{\substack{p \leq q^{r} \\ p \equiv 1(\bmod q)}} \frac{\log p}{p-1} .
$$

1) For all $C>0$ we have $E_{2}(q)=O_{C}(\log \log q)$, with at most $O\left(\frac{\pi(x)}{(\log x)^{C}}\right)$ exceptions $q \leq x$.

2) Assuming EH, we have for fixed $\varepsilon>0$ and $C>0$ that $E_{1+\varepsilon}(q)=$ $O_{C, \varepsilon}(\log \log q)$, with at most $O\left(\frac{\pi(x)}{(\log x)^{C}}\right)$ exceptions $q \leq x$.

3) Assuming ERH, we have $E_{2}(q)=O(\log \log q)$.

Before we consider how the large the prime sum in (17) with $0<$ $r \leq 2$ can be, we remark that it is usually small. 
Proposition 2 (Ford et al. [7]). Uniformly for $z \geq 2, \delta>0$ and $0<\varepsilon \leq 1$, the number of primes $q \leq x$ for which

$$
q \sum_{\substack{p \leq q^{1+\varepsilon} \\ p \equiv 1(\bmod q)}} \frac{\log p}{p-1} \geq \delta \log q
$$

is $O(\varepsilon \pi(x) / \delta)$.

How small $\gamma_{q}$ can be is determined by how large the prime sum in (17) can be.

Proposition 3. There exists an absolute constant $c>0$ such that on a set of primes of natural density 1 we have

$$
-c \log \log q<\frac{\gamma_{q}}{\log q}<(2+\varepsilon) \log q,
$$

with $\varepsilon>0$ arbitrary and fixed.

Under ERH these estimates hold for all primes q large enough.

Proof. On writing the primes $p \equiv 1(\bmod q)$ that satisfy $p \leq q^{2}$ as $a_{1} q+1, \ldots, a_{s} q+1$, and noting that $\mathcal{A}:=\left\{a_{1}, \ldots, a_{s}\right\}$ is an admissible set, we obtain by Proposition 1 that

$$
q \sum_{\substack{p \leq q^{2} \\ p \equiv 1(\bmod q)}} \frac{\log p}{p-1}<2 m(\mathcal{A}) \log q \ll \log q \log \log q .
$$

Now the unconditional statement is obtained on invoking part 1 of Lemma 2.

Under ERH the upper bound is due to Ihara [15] and the lower bound to Badzyan' [3].

In the next section we will see that for $r=2$ the prime sum in 17 can be quite large if we assume HL.

3.1. Assuming HL. Armed with HL and Lemma 2, it is easy to give a conditional disproof of part 1 of Ihara's conjecture.

Theorem 2. Suppose that $H L$ is true and that $\mathcal{A}$ is an admissible set. Then one has

$$
\gamma_{q}<(2-m(\mathcal{A})+o(1)) \log q
$$

for $\gg x \log ^{-\# \mathcal{A}-1} x$ primes $q \leq x$.

\footnotetext{
${ }^{5}$ He assumes GRH. The reproof given in [7, p. 1470] shows that ERH is sufficient.
} 
Proof. Let $a_{1}, \ldots, a_{s}$ be the elements of $\mathcal{A}$. By HL there are infinitely many primes $q$ such that infinitely often $a_{1} q+1, \ldots, a_{s} q+1$ are all prime and in addition $a_{s} q+1 \leq q^{2}$. Then

$$
q \sum_{\substack{p \leq q^{2} \\ p \equiv 1(\bmod q)}} \frac{\log p}{p-1}>q \sum_{i=1}^{s} \frac{\log q}{a_{i} q}=m(\mathcal{A}) \log q .
$$

The proof now easily follows from the part 1 of Lemma 2 with any $C>s$.

A computer calculation gives that $\mathcal{A}=\{a(1), \ldots, a(2088)\}$ satisfies $m(\mathcal{A})>2$, where $a(1), a(2), \ldots$ is the sequence of integers introduced in Section 2.1. We thus obtain the following corollary of Theorem 2.

Corollary 2. Assume HL. Then part 1 of Ihara's conjecture is false for infinitely many primes $q$.

Unconditionally we only have the following result.

Theorem 3 (Ford et al. [7]). We have $\gamma_{964477901}=-0.1823 \ldots$, and so part 1 of Ihara's conjecture is false for at least one prime $q$.

This looks perhaps easy, but was made possible only by a new, fast algorithm developed by the authors of [7] (it requires computation of $L(1, \chi)$ for all characters modulo $q)$. The prime $q=964477901$ has the property that $a q+1$ is prime for $a \in\{2,6,8,12,18,20,26,30,36,56, \ldots\}$. It is easy to approximate the above value of $\gamma_{q}$ by taking a large $x$ in formula (16). The authors of [7] believe that if there is a further $q$ with $\gamma_{q}<0$, then its computation will be hopelessly infeasible.

Since by Theorem 1 one can find admissible $\mathcal{A}$ with $m(\mathcal{A})$ arbitrarily large, we obtain the following result from Theorem 2 .

Theorem 4 (Ford et al. [7]). Assume HL. Then

$$
\lim \inf _{q \rightarrow \infty} \frac{\gamma_{q}}{\log q}=-\infty
$$

Thus, conditionally, $\gamma_{q}$ can be very negative. This happens not frequently since Mourtada and Kumar Murty [27] showed unconditionally that the set of primes $q \leq x$ such that $\gamma_{q} \leq-11 \log q$ is of size $o(\pi(x))$. In Section 5 we speculate how negative $\gamma_{q}$ as a function of $q$ can be.

3.2. Assuming EH (and HL). The prime sum in (17) cannot be too small by Proposition 2, and on invoking the part 2 of Lemma 2 we obtain the following result. 
Theorem 5 (Ford et al. [7]). Assume EH. Let $\varepsilon>0$ be arbitrary. For a density 1 sequence of primes $q$ we have

$$
1-\varepsilon<\frac{\gamma_{q}}{\log q}<1+\varepsilon
$$

This describes the situation for the bulk of the primes. However, if one assumes in addition HL, one can say something about the irregular behaviour.

Theorem 6. Suppose that both $E H$ and $H L$ are true. If $\mathcal{A}$ is an admissible set, then one has

$$
\gamma_{q}=(1-m(\mathcal{A})+o(1)) \log q
$$

for $\gg_{\mathcal{A}} x \log ^{-\# \mathcal{A}-1} x$ primes $q \leq x$.

Sketch of proof. By reasoning as in the proof of Theorem 2, we obtain $\gamma_{q} \leq(1-m(\mathcal{A})+o(1)) \log q$. The reverse inequality is obtained on using sieve methods to find enough primes $q \leq x$ with $q a+1$ prime for $a \in \mathcal{A}$ and not prime for $a \notin \mathcal{A}$ and $a \leq q^{\varepsilon}$, see [7, p. 1465] for details.

Now using that $\overline{\mathcal{M}}=[0, \infty]$ (Corollary 1 ), we obtain the following result.

Theorem 7 (Ford et al. [7]). Assume EH and HL. Then the set

$$
\Theta:=\left\{\frac{\gamma_{q}}{\log q}: q \text { prime }\right\}
$$

is dense in $(-\infty, 1]$.

We propose the following conjecture.

Conjecture 7. Let $\mathcal{A}$ be any admissible set. If $E H$ and $H L$ are both true, then $1-m(\mathcal{A})$ is a limit point of the set $\Theta$.

3.3. Cyclotomic Euler-Kronecker constants on average. Kumar Murty [29] proved unconditionally that

$$
\sum_{Q / 2<q \leq Q}\left|\gamma_{q}\right| \ll(\pi(Q)-\pi(Q / 2)) \log Q .
$$

Fouvry [8] showed that uniformly for $M \geq 3$ one has the equality

$$
\frac{1}{M} \sum_{M / 2<m \leq M}\left|\gamma_{m}\right|=\log M+O(\log \log M),
$$

if one ranges over the integers $m$, rather than the primes $q$. 


\section{The Kummer conjecture: (COnditional) Results}

The orthogonality property of characters gives us

$$
\sum_{\chi(-1)=-1} \log L(s, \chi)=\frac{q-1}{2} \sum_{p^{m} \equiv \pm 1(\bmod q)} \pm \frac{1}{m p^{m s}},
$$

where the latter notation is shorthand for

$$
\sum_{p^{m} \equiv 1(\bmod q)} \frac{1}{m p^{m s}}-\sum_{p^{m} \equiv-1(\bmod q)} \frac{1}{m p^{m s}} .
$$

From Hasse's formula 13 we have that

$$
\log r(q)=\frac{q-1}{2} \lim _{x \rightarrow \infty}\left(\sum_{m \geq 1} \frac{1}{m} \sum_{\substack{p^{m} \leq x \\ p^{m} \equiv \pm 1(\bmod q)}} \pm \frac{1}{p^{m}}\right) .
$$

We denote the limit by $f_{q}$. Note that Kummer's conjecture is equivalent with $f_{q}=o(1 / q)$. Formula (18) should be compared to formula (16). As in that formula, one tries to choose $x$ as small as possible so that the resulting error is still reasonable. In doing so, also here BombieriVinogradov theorem and Brun-Titchmarsh inequality come into play. The main contribution to $f_{q}$ comes from the term with $m=1$. Taking all this into account, Granville [11] showed that if Kummer's conjecture is true, then for every $\delta>0$ we must have

$$
\sum_{\substack{p \leq q 1+\delta \\ p \equiv \pm 1(\bmod q)}} \pm \frac{1}{p}=o\left(\frac{1}{q}\right)
$$

for all but at most $\ll x / \log ^{3} x$ primes $q \leq x$.

Using this approach Granville showed that

$$
1 / c \leq r(q) \leq c
$$

for a positive proportion $\rho(c)$ of primes $p \leq x$, where $\rho(c) \rightarrow 1$ as $c \rightarrow \infty$. Ram Murty and Yiannis Petridis [28] improved this as follows.

Theorem 8. There exists a positive constant c such that for a sequence of primes with natural density 1 we have

$$
c^{-1} \leq r(q) \leq c .
$$

If $E H$ is true, then we can take $c=1+\varepsilon$ for any fixed $\varepsilon>0$.

Thus Ram Murty and Yiannis Petridis showed that a weaker version of Kummer's conjecture holds true. Yet, if both EH and HL are true, Kummer's conjecture itself is false and, moreover, we have the following much stronger result. 
IRREGULAR BEHAVIOUR OF TWO CYCLOTOMIC FIELD INVARIANTS 15

Theorem 9 (Granville [11]). Put

$$
\Omega=\{r(q): q \text { is prime }\} \text {. }
$$

Assume both $H L$ and $E H$. Then the sequence $\Omega$ has $[0, \infty]$ as set of limit points.

This result follows from Corollary 1 and the following.

Theorem 10. If $E H$ and $H L$ are both true, then, for any admissible set $\mathcal{A}$, the numbers $e^{m(\mathcal{A}) / 2}$ and $e^{-m(\mathcal{A}) / 2}$ are both limit points of $\Omega$.

\section{THE LOG LOG LOG DEVIL UNLEASHED}

Regarding the extremal behaviour of $r(q)$ and $\gamma_{q} / \log q$, we enter the realm of speculation, following Granville [11, Section 9].

Speculation 1 (Granville [1]]). For all primes q, we have

$$
(-1+o(1)) \log \log \log q \leq 2 \log r(q) \leq(1+o(1)) \log \log \log q .
$$

These bounds are best possible in the sense that there exist two infinite sequences of primes for which the lower, respectively upper bound are attained.

The same line of thought for $\gamma_{q}$ gives rise to the following speculation.

Speculation 2. For all primes $q$, we have

$$
\frac{\gamma_{q}}{\log q} \geq(-1+o(1)) \log \log \log q \text {. }
$$

The bound is best possible in the sense that there exists an infinite sequence of primes for which the bound is attained.

We will now sketch the motivation for these two speculations and do this in parallel, to bring out the analogy in the reasoning more clearly. The speculations require the assumption that primes are both more regularly and more irregularly distributed than can be currently established.

For convenience let us write $L_{2}=\log \log q$ and $L_{3}=\log \log \log q$. We assume that there exists an absolute constant $A>0$ for which we can take $x=q(\log q)^{A}$ in (16), such that the estimate

$$
\gamma_{q}=\log q-q \sum_{\substack{p \leq q(\log q) A \\ p \equiv 1(\bmod q)}} \frac{\log p}{p-1}+E(q),
$$

with $E(q)=o\left(L_{3} \log q\right)$ holds true. Now note that

$$
\sum_{\substack{p \leq q(\log q)^{A} \\ p \equiv 1(\bmod q)}} \frac{\log p}{p-1}=\sum_{\substack{p \leq q(\log q)^{A} \\ p \equiv 1(\bmod q)}} \frac{\log q}{p}\left(1+O_{A}\left(\frac{L_{2}}{\log q}\right)\right),
$$


where we used that the $\log p$ appearing on the left hand side of $(22)$ satisfies $\log p=\log q+O_{A}\left(L_{2}\right)$. Combining (22) and (21) then yields

$$
\frac{\gamma_{q}}{\log q}=1-\sum_{\substack{2 q-1 \leq p \leq q(\log q)^{3} \\ p \equiv 1(\bmod q)}} \frac{q}{p}\left(1+O_{A}\left(\frac{L_{2}}{\log q}\right)\right)+\frac{E(q)}{\log q} .
$$

Granville [11, p. 335] makes some speculations about the distribution of prime numbers that would ensure that one can go down to $x=q(\log q)^{3}$ in the Kummer problem and lead t $d^{6}$

$$
\log r(q)=\frac{q-1}{2} \sum_{\substack{p \leq q(\log q)^{3} \\ p \equiv \pm 1(\bmod q)}} \pm \frac{1}{p}+O\left(\frac{1}{\sqrt{\log q}}\right) .
$$

By the Brun-Titchmarsh theorem there exists a constant $c>0$ such that for all $x \geq 2 q-1$ we have

$$
\max \{\pi(x ; q,-1), \pi(x ; q, 1)\} \leq c \frac{x}{(q-1) \log (x / q)} .
$$

Using this it is easy to deduce that

$$
\sum_{\substack{2 q+1 \leq p \leq q(\log q) \\ p \equiv 1(\bmod q)}} \frac{1}{p} \leq \frac{c}{q}\left(L_{3}+O_{A}(1)\right) .
$$

Combining this estimate with 23 gives

$$
\frac{\gamma_{q}}{\log q} \geq 1-c L_{3}+O_{A}(1)+\frac{E(q)}{\log q}
$$

Similarly, combining (25) with $A=3$ and (24) yields

$$
\log r(q) \leq c L_{3} / 2+O(1) \text {. }
$$

It follows from (24) that

$$
\log r(q) \geq-\frac{q-1}{2} \sum_{\substack{2 q-1 \leq p \leq q(\log q)^{3} \\ p \equiv-1(\bmod q)}} \frac{1}{p}+O\left(\frac{1}{\sqrt{\log q}}\right),
$$

and a similar argument as before now yields

$$
\log r(q) \geq-c L_{3} / 2+O(1) .
$$

Montgomery and Vaughan [24] have shown that we may take $c=2$ and it is conjectured that one may take $c=1+o(1)$. If this is so, then combining (27) with (28) yields (19). Likewise, (26) gives rise to the lower bound (20).

The final step is to argue why the bounds $(19)$ and $(20)$ are best possible. We will only do so for the easier case of the bound (20). We

\footnotetext{
${ }^{6}$ Having the larger error term $o\left(L_{3}\right)$ would also suffice for our purposes.
} 
assume the Hardy-Littlewood conjecture in a stronger form, namely in its original asymptotic form. Then one can argue that for any admissible set $\mathcal{A}$ with elements $\leq z$, we only find enough primes $q$ for which $p=q a+1$ is prime for all $a \in \mathcal{A}$ if $q>z^{10 z}$ and $z$ is large enough. This in combination with the part 1 of Proposition 1 then suggests that there are infinitely many primes $q$ for which

$$
\sum_{\substack{2 q+1 \leq p \leq q(\log q) A \\ p \equiv 1(\bmod q)}} \frac{1}{p} \geq(1-\varepsilon) \frac{L_{3}}{q} .
$$

This estimate, together with $(23)$ and the already obtained lower bound (20), then finishes our argumentation.

These two speculations taken together imply the following weaker one.

Speculation 3. There exists a function $g(q)$ such that

$$
\lim \inf _{q \rightarrow \infty} \frac{\gamma_{q}}{g(q) \log q}=2 \lim \inf _{q \rightarrow \infty} \frac{\log r(q)}{g(q)}<0 .
$$

In case $g(q)$ is not the $\log \log \log$ devil from the title, it is certainly a close cousin!

Comparison of Conjecture 7 and Theorem 10 suggests that $\gamma_{q} / \log q$ and $1-2|\log r(q)|$ behave similarly, which is consistent with the three speculations presented in this section.

\section{Prospect}

6.1. Polymath. Recent progress on gaps between primes allows one to meet the challenge below for some $C>0$. Indeed, according to James Maynard [23], recent results allow one to take $C=1 / 246$.

Challenge 1. Find a set $\mathcal{A}=\left\{a_{1}, \ldots, a_{s}\right\}$ such that provably for some $B>0$ there are $\gg x / \log ^{B} x$ primes $q \leq x$ such that $a_{1} q+1, \ldots, a_{s} q+1$ are all prime and, in addition,

$$
\sum_{i=1}^{s} \frac{1}{a_{i}} \geq C
$$

with $C$ as large as possible.

Conjecturally $C$ can be taken arbitrarily large, cf. Theorem 1 .

Proposition 4. If one meets the challenge for any $C>2$, then there are $\gg x / \log ^{B} x$ primes $q \leq x$ for which part 1 of Ihara's conjecture is false and, moreover, $\gamma_{q}<(2-C) \log q$.

Proof. Similar to that of Theorem 2. 
6.2. Kummer for arbitrary cyclotomic fields. It is not difficult to formulate a generalized Kummer conjecture, where instead of the primes we range over the integers. Goldstein [10] established that, as $r$ tends to infinity and $q$ is fixed, we have

$$
\log h_{1}\left(q^{r}\right) \sim \frac{r}{4}\left(1-\frac{1}{q}\right) q^{r} \log q .
$$

Myers [30] obtained some results along the lines of Ram Murty and Petridis [28]. Fouvry [8] determined the average order of $\left|\gamma_{m}\right|$ (see Section 3.3. Quite likely further results can be obtained, e.g., it is perhaps possible to find explicit composite integers $m$ for which $\gamma_{m}<0$.

6.3. Strengthening the analogy (Moree and Saad Eddin [26]). Comparison of (8) and (13) suggests that one can expect an even closer analogy between $r(q)$ and the difference

$$
\gamma_{q}-\gamma_{q}^{+}=\sum_{\chi(-1)=-1} \frac{L^{\prime}(1, \chi)}{L(1, \chi)},
$$

which results on subtracting $(10)$ from $(8)$. In particular, it is to be expected that $\gamma_{q}-\gamma_{q}^{+}$will display, like $\log r(q)$, a more symmetric behaviour around the origin than $\gamma_{q}$ does. Also recall that $r(q)$ and $\gamma_{q}-\gamma_{q}^{+}$both appear in the Taylor series (14).

Acknowledgment. I like to thank James Maynard for pointing out that one can take $C=1 / 246$ in Challenge 1. Furthermore, I am grateful to Alexandru Ciolan, Sumaia Saad Eddin and Alisa Sedunova for proofreading and help with editing an earlier version. Ignazio Longhi and the referee kindly pointed out some disturbing typos.

The similarity between Kummer's and Ihara's conjectures was pointed out by Andrew Granville after a talk given by Kevin Ford on [7]. At that point the authors of [7] had independently obtained Theorem 1 , but not Granville's Proposition 1, the latter being precisely the result used by Granville to unleash the log log log devil. Once at the loose, it created havoc also among the Euler-Kronecker constants.

\section{REFERENCES}

[1] N.C. Ankeny and S. Chowla, The class number of the cyclotomic field, Proc. Nat. Acad. Sci. U. S. A. 35 (1949), 529-532.

[2] N.C. Ankeny and S. Chowla, The class number of the cyclotomic field, Canadian J. Math. 3 (1951), 486-494.

[3] A.I. Badzyan, The Euler-Kronecker constant, Mat. Zametki 87 (2010), 45-57. English Translation in Math. Notes 87 (2010), 31-42. 
[4] E.S. Croot, III and A. Granville, Unit fractions and the class number of a cyclotomic field, J. London Math. Soc. (2) 66 (2002), 579-591.

[5] K. Debaene, The first factor of the class number of the $p$-th cyclotomic field, Arch. Math. (Basel) 102 (2014), 237-244.

[6] P. Erdős, On a problem of S. Golomb7] J. Austral. Math. Soc. 2 (1961/1962), $1-8$.

[7] K. Ford, F. Luca and P. Moree, Values of the Euler $\phi$-function not divisible by a given odd prime, and the distribution of Euler-Kronecker constants for cyclotomic fields, Math. Comp. 83 (2014), 1447-1476.

[8] É. Fouvry, Sum of Euler-Kronecker constants over consecutive cyclotomic fields, J. Number Theory 133 (2013), 1346-1361.

[9] G. Fung, A. Granville and H.C. Williams, Computation of the first factor of the class number of cyclotomic fields, J. Number Theory 42 (1992), 297-312.

[10] L.J. Goldstein, On the class numbers of cyclotomic fields, J. Number Theory 5 (1973), 58-63.

[11] A. Granville, On the size of the first factor of the class number of a cyclotomic field, Inv. Math. 100 (1990), 321-338.

[12] Y. Hashimoto, Euler constants of Euler products, J. Ramanujan Math. Soc. 19 (2004), 1-14.

[13] Y. Hashimoto, Y. Iijima, N. Kurokawa and M. Wakayama, Euler's constants for the Selberg and the Dedekind zeta functions, Bull. Belg. Math. Soc. Simon Stevin 11 (2004), 493-516.

[14] H. Hasse, Über die Klassenzahl Abelscher Zahlkörper, Mathematische Lehrbücher und Monographien, Band I, Berlin, Akademie-Verlag, 1952.

[15] Y. Ihara, On the Euler-Kronecker constants of global fields and primes with small norms, in V. Ginzburg, ed., Algebraic Geometry and Number Theory: In Honor of Vladimir Drinfeld's 50th Birthday, Progress in Mathematics 850, Birkhäuser Boston, Cambridge, MA, 2006, 407-451.

[16] Y. Ihara, The Euler-Kronecker invariants in various families of global fields, Proc. of AGCT 2005 (Arithmetic Geometry and Coding Theory 10), Ed. F. Rodier et al., Séminaires et Congrès 21 (2009), 79-102.

[17] Y. Ihara, On " $M$-functions" closely related to the distribution of $L^{\prime} / L$-values, Publ. Res. Inst. Math. Sci. 44 (2008), 893-954.

[18] Y. Ihara, V.K Murty and M. Shimura, On the logarithmic derivatives of Dirichlet $L$-functions at $s=1$, Acta Arith. 137 (2009), 253-276.

[19] E.E. Kummer, Mémoire sur la théorie des nombres complexes composées de racines de l'unité et des nombres entiers, J. Math. Pures Appl. 16 (1851), 377-498 (1851); Collected Works, Vol. I., pp. 363-484.

[20] J.C. Lagarias, Euler's constant: Euler's work and modern developments, Bull. Amer. Math. Soc. (N.S.) 50 (2013), 527-628.

[21] S. Lang, Cyclotomic fields I and II, Combined second edition, Graduate Texts in Mathematics 121, Springer-Verlag, New York, 1990.

[22] J.M. Masley and H.L. Montgomery, Cyclotomic fields with unique factorization, J. Reine Angew. Math. 286/287 (1976), 248-256.

[23] J. Maynard, E-mail to author, 04/14/2014.

[24] H.L. Montgomery and R.C. Vaughan, The large sieve, Mathematika 20 (1973), $119-134$.

\footnotetext{
${ }^{7}$ The title of the published paper has "G. Golomb", a misprint
} 
[25] P. Moree, Counting numbers in multiplicative sets: Landau versus Ramanujan, Mathematics Newsletter 21, no. 3 (2011), 73-81 (arXiv:1110.0708).

[26] P. Moree and S. Saad Eddin, Euler-Kronecker constants for maximal real cyclotomic fields and Kummer's conjecture, in preparation.

[27] M. Mourtada and V.K. Murty, On the Euler Kronecker constant of a cyclotomic field, II. SCHOLAR -a scientific celebration highlighting open lines of arithmetic research, 143-151, Contemp. Math. 655, Centre Rech. Math. Proc., Amer. Math. Soc., Providence, RI, 2015.

[28] M.R. Murty and Y.N. Petridis, On Kummer's conjecture, J. Number Theory 90 (2001), 294-303.

[29] V.K. Murty, The Euler-Kronecker constant of a number field, Ann. Sci. Math. Québec 35 (2011), 239-247.

[30] M.J.R. Myers, A generalised Kummer's conjecture, Glasg. Math. J. 52 (2010), 453-472.

[31] W. Narkiewicz, Elementary and analytic theory of algebraic numbers. Second edition. Springer-Verlag, Berlin; PWN-Polish Scientific Publishers, Warsaw, 1990.

[32] J.-C. Puchta, On the class number of $p$-th cyclotomic field, Arch. Math. (Basel) 74 (2000), 266-268.

[33] M.A. Shokrollahi, Relative class number of imaginary abelian fields of prime conductor below 10000, Math. Comp. 68 (1999), 1717-1728.

[34] C.L. Siegel, Zu zwei Bemerkungen Kummers, Nachr. Akad. Wiss. Gttingen Math.-Phys. Kl. II 6 (1964), 51-57; Collected Works III, 438-442.

[35] M.A. Tsfasman, Asymptotic behaviour of the Euler-Kronecker constant, in V. Ginzburg, ed., Algebraic Geometry and Number Theory: In Honor of Vladimir Drinfeld's 50th Birthday, Progress in Mathematics, Vol. 850, Birkhäuser Boston, Cambridge, MA, 2006, 453-458.

[36] L.C. Washington, Introduction to cyclotomic fields, Graduate Texts in Mathematics 83, Springer-Verlag, New York, 1982.

MaX-Planck-Institut Für Mathematik, Vivatsgasse 7, D-53111 Bonn, GERMANY

E-mail address: moree@mpim-bonn.mpg.de 